\title{
Bartonella henselae associated uveitis and HLA-B27
}

\author{
F T Kerkhoff, A Rothova
}

\begin{abstract}
Aim-To investigate the frequency of HLA-B27 in patients with presumed Bartonella henselae associated uveitis and to describe the clinical characteristics of HLA-B27 positive patients with uveitis and presumed ocular bartonellosis (POB).

Methods-The diagnosis of POB was considered in 19 patients with unexplained uveitis (except for the HLA-B27 association) and high positive IgG (titre $\geqslant 1: 900$ ) and/or IgM (titre $\geqslant 1: 250$ ) antibodies against $B$ henselae. In addition to $B$ henselae serology and HLA-B27 typing, all patients underwent an extensive standard diagnostic screening procedure for uveitis and in all cases the results were within the normal limits. The control group consisted of 25 consecutive patients with panuveitis and negative $B$ henselae serology.
\end{abstract}

Results-HLA-B27 was positive in six of the 19 patients $(32 \%)$ with $P O B$ in contrast to the $4 \%$ frequency of HLA-B27 in the control group $(p=0.03)$ and $8 \%$ prevalence of HLA-B27 in the Dutch population $(p=0.003)$. At the time of positive Bartonella serological testing five of six HLAB27 positive patients with $P O B$ had severe posterior segment involvement with papillitis, macular oedema, and vitreitis. The duration of intraocular inflammatory activity was more than 6 months in five HLA-B27 positive patients. Although four of the six HLA-B27 positive patients had previous recurrent attacks of acute anterior uveitis, the clinical presentation at the time of positive Bartonella serology differed, as illustrated by the involvement of the posterior segment and chronic course of the ocular disease.

Conclusions-The frequency of HLA-B27 in patients with uveitis and serological characteristics of acute infection with $B$ henselae is higher than in the general Dutch population. The findings of this study also suggest a relation between infection with Bartonella species and HLA-B27.

(Br f Ophthalmol 2000;84:1125-1129)

Ophthalmology, FC

Donders Institute,

University Hospital,

3508 GA Utrecht,

Netherlands

F T Kerkhoff

A Rothova

Correspondence to:

Dr F T Kerkhoff

fkerkho@oogh.azu.nl

Accepted 3 May 2000 understood but, apart from the immunogenetic background, other factors such as envi- ronmental agents may play an important part in the development of the disease. ${ }^{34}$ Bacterial agents such as Gram negative bacteria (Klebsiella, Proteus, Salmonella, Yersinia, Shigella, and Campylobacter) and Chlamydia have been implicated in the development of HLA-B27 associated diseases. ${ }^{5-9}$ The frequency of HLA-B27 varies in different races, being $4-13 \%$ in the white population, $2-3 \%$ in the American black population, and almost absent in some genetically unmixed native populations. ${ }^{10}{ }^{11}$ In surveys of uveitis the frequency of HLA-B27 associated acute anterior uveitis also varies among the different races; in a Dutch study of 865 consecutive patients with uveitis Rothova $e t a l^{12}$ found HLA-B27 acute anterior uveitis in 17\% whereas Derhaag et $a l^{13}$ reported HLA-B27 in $50 \%$ of Dutch patients with the condition.

Bartonella henselae is a Gram negative rod and was identified as the organism causing cat scratch disease (CSD). ${ }^{14}$ Ocular involvement in the course of $B$ henselae infection has been reported to be the cause of Parinauld's oculoglandular syndrome, uveitis, and neuroretinitis. $^{15} 16$ The association of HLA B27 and $B$ henselae associated uveitis was noted in two earlier reports. ${ }^{17} 18$

We have investigated the frequency of HLAB27 in presumed ocular bartonellosis and report the clinical features and laboratory data of these patients.

\section{Patients and methods}

Nineteen patients with uveitis and high positive IgG and/or IgM antibody titres for $B$ henselae seen in the F C Donders Institute, University Medical Center between January 1994 and December 1997 were included in the study. Five patients were selected from a cohort of 138 consecutive patients with uveitis seen in our institute in 1994; in this cohort $B$ henselae serological testing was performed for all patients as part of an earlier study on ocular bartonellosis. ${ }^{16}$ Fourteen additional patients were identified between January 1995 and December 1997; at this time the determination of antibodies against $B$ henselae was not a routine procedure so the tests were predominantly performed in patients with clinical features consistent with the diagnosis of ocular bartonellosis (mostly patients with papillitis and panuveitis or posterior uveitis of unknown cause). Anatomical classification of uveitis was performed according to the criteria defined by the International Uveitis Study Group. ${ }^{19} \mathrm{~Pa}-$ tients with neuroretinitis were classified separately from those with posterior uveitis. Neuroretinitis was characterised by swelling of the optic disc and peripapillary oedema, eventually 
combined with macular oedema and hard exudates in the macular area (macular star).$^{20}$ In addition to $B$ henselae serological tests and HLA-B27 typing, all patients underwent a standard diagnostic screening procedure for uveitis which consisted of erythrocyte sedimentation rate, red and white blood cell counts, serum angiotensin converting enzyme levels, syphilis serology, and chest radiography. Fluorescein angiography was performed in 19 patients and visual field testing in nine. The control group consisted of 25 consecutive patients with panuveitis and negative $B$ henselae serology.

Antibodies against $B$ henselae were determined by enzyme immunoassay (EIA) as described by Bergmans et al and the cut off values for positive serology were $\geqslant 1: 900$ for IgG-EIA and $\geqslant 1: 250$ for IgM-EIA. ${ }^{21}$ The serum samples for the determination of $B$ henselae antibodies were in all cases obtained during the active stage of the ocular disease. On the basis of positive $B$ henselae serological findings, 19 patients were considered to have presumed ocular bartonellosis (POB). A $\chi^{2}$ test was used for the statistical analysis to compare the prevalence of HLA-B27 in the control group and in the Dutch population with the frequency of HLA-B27 in patients with POB. ${ }^{22}$ These 19 patients were subdivided into HLAB27 positive and negative groups and their clinical characteristics were compared.

\section{Results}

The general characteristics of the 19 patients with POB are shown in Table 1 . The mean age of the patients (seven men, 12 women) was 47 years (range 9-79). Fifteen patients were white, two were of Mediterranean origin, one was black, and one was from the Far East. The follow up period since the diagnosis of POB in all patients was at least 1 year (range 12-46 months). Of the 19 patients with POB six $(32 \%)$ were HLA-B27 positive, which was significantly higher than the control group in which only one of $25(4 \%)$ was HLA-B27 positive $(\mathrm{p}=0.03)$ and the prevalence of $8 \%$ in the Dutch population $(\mathrm{p}=0.003)$.

The diagnosis of POB was based in 14 patients on serum IgG-EIA titres against $B$ henselae of $\geqslant 1: 900$ and in five patients on serum IgM-EIA titres against $B$ henselae of $\geqslant 1: 250$ (Table 1 ). Of the HLA-B27 positive patients one was $\operatorname{IgM}$ and five were $\operatorname{IgG}$

Table 1 General characteristics of HLA-B27 positive and negative patients with presumed ocular bartonellosis (POB)

\begin{tabular}{lll}
\hline General characteristics & $\begin{array}{l}\text { HLA-B27 } \\
\text { positive (n=6) }\end{array}$ & $\begin{array}{l}\text { HLA-B27 } \\
\text { negative }(n=13)\end{array}$ \\
\hline Mean (range) age (years) & $45(28-55)$ & $49(15-69)$ \\
Race & 3 & 12 \\
$\quad$ White & 3 & 1 \\
$\quad$ Non-white & 4 & 9 \\
Animal exposures & 0 & 3 \\
Systemic CSD & \\
Mean (range) follow up & & \\
$\quad$ after diagnosis of POB & & $30(14-46)$ \\
$\quad$ (months) & $27(12-46)$ & 10 \\
B henselae & & 4 \\
$\quad$ EIA-IgG $\geqslant 1: 900$ & 5 & \\
EIA-IgM $\geqslant 1: 250$ & 1 &
\end{tabular}

$\mathrm{CSD}=$ cat scratch disease.
Table 2 Ocular features of HLA-B27 positive and negative patients with presumed ocular bartonellosis (POB)

\begin{tabular}{lll}
\hline Ocular features & $\begin{array}{l}\text { HLA-B27 } \\
\text { positive }(n=6)\end{array}$ & $\begin{array}{l}\text { HLA-B27 } \\
\text { negative }(n=13)\end{array}$ \\
\hline $\begin{array}{l}\text { Posterior segment } \\
\text { involvement }\end{array}$ & 5 & 13 \\
Bilateral disease & 2 & 10 \\
Aqueous cells & 6 & 11 \\
Posterior synechiae & 4 & 5 \\
Vitreous cells & 5 & 12 \\
Macular oedema & 5 & 8 \\
Papillitis & 5 & 12 \\
Retinal lesions & 0 & 4 \\
Vasculitis & 1 & 4 \\
Initial visual acuity of $<0.1$ & $4 / 8^{\star}$ & $2 / 22$ \\
Final visual acuity of $<0.1$ & $1 / 8$ & $1 / 22$ \\
\hline
\end{tabular}

«Significantly more affected eyes with initial visual acuity of $<0.1$ than in HLA-B27 negative group ( $\mathrm{p}=0.03)$.

positive; of the HLA-B27 negative patients four were $\operatorname{IgM}$ and 10 were $\operatorname{IgG}$ positive (Table 1). Exposure to cats or dogs before the ocular disease was reported in nine HLA-B27 negative and three HLA-B27 positive patients. Only three HLA-B27 negative patients had systemic symptoms characteristic of CSD - that is, unexplained fever, malaise, and lymphadenopathy. One HLA-B27 positive patient was diagnosed with ankylosing spondylitis (14 months after the onset of ocular disease). Two HLA-B27 negative patients were positive for anticardiolipin antibodies, one of whom developed general malaise with recurrent episodes of fever and arthralgia two years after the ocular symptoms.

The ocular features in the HLA-B27 positive and negative groups did not differ significantly (Table 2). In the whole series one patient presented with anterior uveitis (HLA-B27 positive), 12 with panuveitis (four HLA-B27 positive, eight HLA-B27 negative), and seven with neuroretinitis (one HLA-B27 positive, six HLA-B27 negative, Table 2). Unilateral uveitis was more frequent in the HLA-B27 positive group and bilateral uveitis in the HLA-B27 negative group. Optic nerve involvement was detected on fluorescein angiography in five HLA-B27 positive patients and 12 HLA-B27 negative patients. Macular oedema was observed in five HLA-B27 positive patients (one had typical macular star) and eight negative patients. In four HLA-B27 negative patients small peripheral multifocal chorioretinal lesions were detected. One of the HLA-B27 positive patients had a large atrophic area in the peripheral retina of the affected eye.

Five of the HLA-B27 positive POB patients had severe posterior segment involvement with inflammatory cells in the vitreous, papillitis, and macular oedema at the time of positive Bartonella serology. In all five the inflammatory activity in the posterior segment persisted for more than 6 months. Four of the HLA-B27 positive POB patients had previous recurrent acute anterior uveitis but, at the time of $B$ henselae testing, presented with involvement of the posterior segment. Two of these patients subsequently developed chronic panuveitis (duration more than 12 months). In the two patients without previous acute anterior uveitis, one developed chronic anterior uveitis after the initial presentation with panuveitis and 
positive $B$ henselae serology and the remaining patient had isolated neuroretinitis and remained without recurrences during 30 months of follow up.

The visual acuity at presentation was lower in the HLA-B27 positive group than in the HLA-B27 negative group $(p=0.03)$ and was predominantly caused by media opacities such as intraocular inflammatory cells and vitreous floaters (Table 2). The initial visual acuity of less than 20/200 was found for six of 30 affected eyes (four eyes of HLA-B27 positive patients). The final visual acuity did not differ between the HLA-B27 positive and negative groups. At 12 months follow up two of the 30 affected eyes had visual acuity of less than 20/200 (one HLA-B27 positive and one negative). In one HLA-B27 positive patient with panuveitis visual acuity decreased to light perception because of an exudative retinal detachment, and one HLA-B27 negative patient with neuroretinitis had a follow up visual acuity of counting fingers caused by optic nerve atrophy. Persistent visual field defects were noted for one of four eyes of HLA-B27 positive patients and for 10 of 14 eyes of HLA-B27 negative patients.

Fifteen patients were treated with antibiotics, three with clarithromycin, three with ciprofloxacin hydrochloride, and nine with doxycycline and rifampicin. Before the antibiotic treatment all 15 patients received periocular or systemic treatment with corticosteroids. In eight of the 15 patients the intraocular inflammation subsided after antibiotic treatment. No significant differences in treatment efficacy were observed between the HLA-B27 positive and negative patients or between the patients with various presentations of intraocular inflammation.

CASE REPORTS

Case 1

A 37 year old woman was referred to our uveitis clinic because of a bilateral but asymmetrical panuveitis of one year's duration for which she was symptomatically treated with systemic corticosteroids without success (Fig 1). During the corticosteroid treatment she developed an exudative retinal detachment which resolved after treatment was withdrawn. Her ophthalmic history revealed recurrent HLA-B27 associated acute anterior uveitis over 12 years. On presentation her visual acuity was $20 / 100$ in the right eye and 20/20 in the left eye. The anterior chambers were clear; in the right eye old posterior synechiae were noted. In the vitreous of the right eye inflammatory cells and infiltrates were visible. Funduscopy revealed a hyperaemic swollen optic disc (Fig 1) in the right eye and in the retinal periphery an area of chorioretinal atrophy was noted without signs of retinal inflammation or vasculitis. Intraocular pressure in the right eye was normal. The left eye revealed no abnormalities except for a hyperaemic but not swollen optic disc and an intraocular pressure of $48 \mathrm{~mm} \mathrm{Hg}$. The serum IgG-EIA titre for $B$ henselae was 1:1200. A diagnosis of POB was made and the patient was subsequently treated with doxycycline and

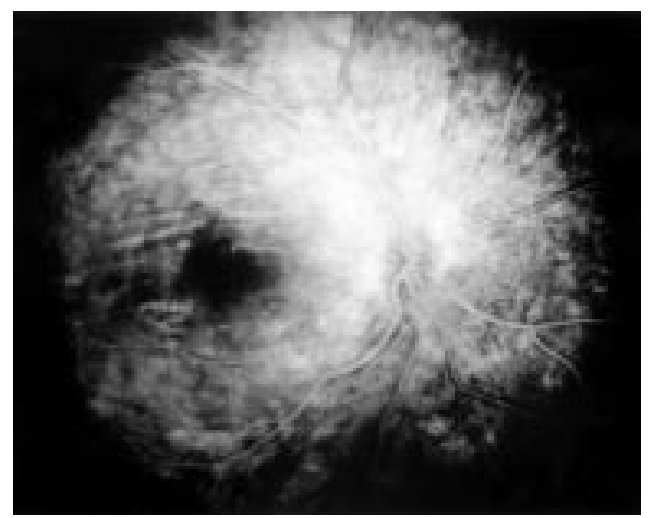

Figure 1 Fluorescein angiogram of the right eye (case 1) showing an extremely hyperfluorescent optic nerve and peripapillary leakage of fluorescein.

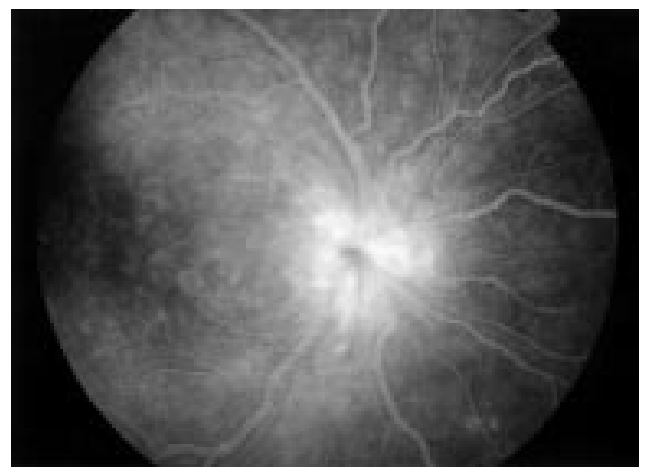

Figure 2 Fluorescein angiogram of the right eye (case 2) showing a hyperfluorescent optic nerve and macular oedema.

rifampicin. The raised intraocular pressure in the left eye was symptomatically treated. However, after a short period of improvement, panuveitis and exudative retinal detachment in the right eye recurred and optic disc pallor developed. The visual acuity of the right eye decreased to light perception.

\section{Case 2}

A 48 year old woman was referred to our uveitis clinic because of a severe unilateral panuveitis in the right eye (Fig 2). Her ophthalmic history revealed bilateral cataract extraction at the age of 41 years. The cause of her cataract was not known and there was no family history to account for the development of cataract at an early age. Detailed information about the ocular surgery was not available; the patient had not experienced symptoms of uveitis and received no ophthalmic treatment before her cataract surgery. Five years after the cataract surgery the patient developed a unilateral panuveitis with a swollen optic nerve in the right eye. The visual acuity in the right eye at that time was 20/200. Raised serum IgM-EIA for $B$ henselae in a titre of 1:260 was found and the patient tested positive for HLA-B27. She was subsequently treated with doxycycline and rifampicin and visual acuity improved to 20/60. The panuveitis subsided, but 2 years after the antibiotic treatment chronic anterior uveitis developed in the right eye. 


\section{Discussion}

This report presents 19 patients with high positive antibody titres against $B$ henselae of which six patients $(32 \%)$ were HLA-B27 positive. The frequency of HLA-B27 in patients with $\mathrm{POB}$ was significantly higher than in the control group of patients with panuveitis and in the general Dutch population. ${ }^{22}$ Five of these HLA-B27 positive patients with serological characteristics of $B$ henselae infection presented with uveitis characterised by severe posterior segment involvement. Furthermore, in four HLA-B27 positive patients with a previous history of acute anterior uveitis the clinical features at the time of positive $B$ henselae serology differed from the previous uveitis attacks, which were restricted to the anterior segment. These findings suggest that infection with Gram negative Bartonella may alter or even initiate the course of HLA-B27 associated uveitis.

Although acute anterior uveitis is the most characteristic presentation of HLA-B27 associated ocular disease, our study included only one patient with acute anterior uveitis at the time of positive $B$ henselae serology. His positive results were detected as part of the serological analysis of consecutive subjects in a large series with uveitis. ${ }^{17}$ Uveitis with involvement of the posterior segment has been regularly noted in various HLA-B27 associated diseases such as ankylosing spondylitis, Reiter's syndrome, inflammatory bowel disease, and psoriatic arthritis. ${ }^{23}$ The frequency of posterior segment involvement in HLA-B27 associated uveitis is not precisely known. Linssen et al found posterior segment involvement in one of 119 patients who initially presented with HLA-B27 positive acute anterior uveitis. ${ }^{24}$ Rodriguez et al reported a prevalence of $17.4 \%$ posterior segment involvement in HLA-B27 associated uveitis. ${ }^{25}$ The cause of this discrepancy is not known, but the high prevalence of posterior segment involvement in the latter report may be caused by different inclusion criteria and may also reflect the referral bias of a large tertiary care centre. The high frequency of posterior segment involvement in this study might be caused by the selection criteria to test for $B$ henselae antibodies. Vitreitis and papillitis were the most frequently observed posterior segment features in our study and similar features were observed in the study by Rodriguez et al. ${ }^{25}$ The clinical presentation of posterior segment involvement in our study was similar to that attributed to bartonellosis and did not differ between the HLA-B27 positive and negative groups. In HLA-B27 positive patients with posterior segment inflammation testing for Bartonella could be considered.

Two earlier studies reported the association of HLA-B27 associated uveitis with a positive Bartonella serological test in which one, an HLA-B27 positive patient, was described with Parinauld's oculoglandular syndrome (positive $B$ henselae serology) and the subsequent development of acute anterior uveitis. ${ }^{17}{ }^{18}$ Although the development of arthritis was reported during the course of cat scratch disease, no association was noted with HLA-B27. ${ }^{26}$ Our HLAB27 positive patients with uveitis and high $B$ henselae serological titres had no rheumatological symptoms except for one who was diagnosed with ankylosing spondylitis. One HLAB27 negative patient developed general malaise and arthralgia 2 years after the neuroretinitis.

The frequency of HLA-B27 differs among the various racial groups, resulting in a different association between anterior uveitis and HLA-B27. ${ }^{11}{ }^{27}$ In our series three of six HLA-B27 positive patients were not white. This is a remarkable finding for which we do not have an explanation, and it also does not represent the Dutch racial distribution. Infections with $B$ henselae were predominantly observed in children and immunosuppressed patients, whereas infections with $B$ quintana were frequently observed in homeless people but, to our knowledge, a racial predominance of Bartonella infection was not noted. ${ }^{1628} 29$

The pathogenesis of HLA-B27 associated diseases and involvement of Gram negative micro-organisms is not completely understood. Infections with Gram negative bacteria such as Yersinia, Klebsiella, Shigella, Campylobacter, Salmonella, and Chlamydia may be followed by the development of uveitis, ankylosing spondylitis, and reactive arthritis..$^{5-9}$ An experimental study described the development of acute anterior uveitis in rats following systemic infection with Salmonella enteritidis and Yersinia enterocolita. ${ }^{30}$ Raised serum antibodies against Klebsiella were documented in patients with acute anterior uveitis and ankylosing spondylitis. ${ }^{31}$ Data on the presence of Gram negative bacteria in the eyes of patients with HLA-B27 associated acute anterior uveitis are not available. Polymerase chain reaction (PCR) analysis for Gram negative microorganisms in the intraocular fluids of these patients has, to our knowledge, never been documented. However, Klebsiella and Chlamydia antigens have been detected in the synovial fluid of arthritis patients. ${ }^{32}$ So far the presence of Bartonella DNA in the eye has been reported in the retina of one AIDS patient of unknown HLA-B27 haplotype and in the ocular fluids of an immunocompetent patient with neuroretinitis. $^{33} 34$

To clarify the inflammatory disease in HLAB27 positive patients, some authors considered cross reactivity between the bacterial cell wall and the HLA-B27 antigen and indicated molecular similarity in amino acid sequences between HLA-B27 and bacterial proteins. ${ }^{35}$ Others have suggested a specific B27/microbial interaction that modulates the degree of invasion of pathogens into the host cell. ${ }^{36}$ These mechanisms might also apply to the interaction between an HLA-B27 positive host and Gram negative Bartonella microorganisms. Recent studies indicate that other Bartonella subspecies can also be implicated in the pathogenesis of human diseases. ${ }^{37}$ However, serological assays at this time can only differentiate between antibodies against $B$ henselae and $B$ quintana.

The route of infection in bartonellosis presumably consists of a cutaneous lesion after a cat (or dog) scratch or bite, but alternative routes of infection such as insect bites have also 
been suggested. ${ }^{37}$ Infections with Yersinia, Klebsiella, Shigella, Campylobacter, Salmonella, and Chlamydia are usually contracted by the gastrointestinal or urogenital route..$^{5-9} 35$ Increased serum levels of $\operatorname{IgA}$ in patients with acute anterior uveitis and ankylosing spondylitis might support the hypothesis that mucosal infections are involved in the pathogenesis of HLA-B27 associated diseases. ${ }^{35}$

We conclude that the frequency of HLAB27 in patients with uveitis and serological characteristics of acute infection with $B$ henselae is higher than in the general Dutch population. This report suggests a relation between infection with Bartonella species and HLA-B27. The question whether Bartonella infection modifies HLA-B27 associated uveitis or whether HLA-B27 positive patients are more likely to develop ocular disease during Bartonella infection cannot be answered yet. Future studies are needed to evaluate the frequency and the importance of infection with Bartonella species in patients with HLA-B27 associated diseases.

Supported in part by the Dr FP Fisher Foundation, Utrecht, Netherlands.

1 Brewerton DA, Caffrey M, Nicholls A, et al. Acute anterior uveitis and HL-A 27. Lancet 1973;3:994-6.

2 Brewerton DA, Caffrey M, Hart FD, et al. Ankylosing spondylitis and HL-A 27. Lancet 1973;3:904-7.

3 Feltkamp TEW. Are Gram negative bacteria involved in HLA B27 associated uveitis? Br f Ophthalmol 1995;79:718.

Uksila J, Toivanen P. HLA B27 and bacteria. Clin Rheumatol 1996;15:44-7.

5 Blankenberg-Spenkels SHD, Fielder M, Feltkamp TEW, $e$ al. Antibodies to Klebsiella pneumoniae in Dutch patients with ankylosing spondylitis and acute anterior uveitis and to Proteus mirabilis in rheumatoid arthritis. 7 Rheumatol to Proteus mira

6 Mattila L, Leirisalo-Repo M, Koskimies S, et al. Reactive arthritis following an outbreak of Salmonella infection in Finland. Brf Rheumatol 1994;33:1136-41.

7 Mattila L, Granfors K, Toivanen A. Acute anterior uveitis after Yersinia infection. Br f Ophthalmol 1982;66:209-12.

8 Kosunen TU, Kauranen O, Martio J, et al. Reactive arthritis after Campylobacter jejuni enteritis in patients with HLA B27. Lancet 1980;14:1312-3.

9 Wakefield D, Penny R. Cell mediated immune response to chlamydia in anterior uveitis: role of HLA B27. Clin Exp Immunol 1983;51:191-6.

10 Tay-Kearny ML, Schwam BL, Lowder C, et al. Clinical features and associated systemic diseases of HLA B27 uveitis. Am f Ophthalmol 1996;121:47-57.

11 Khan MA. Epidemiology of HLA B27 and arthritis. Clin Rheumatol 1996;15:10-12.

12 Rothova A, Buitenhuis HJ, Meenken C, et al. Uveitis in systemic disease. Br f Ophthalmol 1992;76:137-41.

13 Derhaag PJFM, van der Horst AR, de Waal LP, et al. HLA $\mathrm{B} 27+$ acute anterior uveitis and other antigens of the majo histocompatibility complex. Invest Ophthalmol Vis $\mathrm{Sc}$ 1989;30:2160-4
14 Regnery RL, Olson JG, Perkins BA, et al. Serological respory RL, "Olson JG, Perkins BA, et al. Serological response to "Rochalimaea henselae" antigen

15 Kerkhoff FT, Ossewaarde JM, de Loos WS, et al. Presumed ocular bartonellosis. Br f Ophthalmol 1999;83:270-5.

16 Carithers HA. Oculoglandular disease of Parinaud. Am $\mathcal{J}$ Dis Child 1978;132:1195-200.

17 Rothova A, Kerkhoff FT, Hooft HJ, et al. Bartonella serology for patients with intraocular inflammatory disease. Retina 1998;18:348-55.

18 Rehman SU, Metcalfe TW. Anterior uveitis associated with cat scratch disease. Br f Ophthalmol 1998;82:587-8.

19 Bloch-Michel E, Nussenblatt RB. International Uveitis Study Group recommendations for the evaluation of intraocular inflammatory disease. Am f Ophthalmol 1987; 103:234-5.

20 Gyer DR, D'Amico DJ. Leber's stellate idiopathic neuroretinitis. In: Albert DM, Jacobeic FA, eds. Principles and practice of ophthalmology: clinical practice. Philadelphia: WB praunders, 1994:809-13.

21 Bergmans AMC, Peeters MF, Schellekens JFP, et al. Pitfalls and fallacies of cat scratch disease serology: evaluation of Bartonella henselae-based indirect fluorescence assay and enzyme-linked immunoassay. f Clin Microbiol 1997;35: 1931-7.

22 Derhaag PJFM, de Waal LP, Linssen A, et al. Acute anterior uveitis and HLA-B27 subtypes. Invest Ophthalmol Vis Sci 1988;29:1137-40.

23 Nussenblatt RB, Whitcup SM, Palestine AG, eds. Anterior uveitis. In: Fundamentals. Uveitis fundamentals and clinical practice. St Louis: Mosby, 1996:265-78.

24 Linssen A, Meenken C. Outcomes of HLA B27-positive and HLA B27-negative acute anterior uveitis. $A m$ f Ophthalmol 1995;120:351-61.

25 Rodriguez A, Akova YA, Pedroza-Seres M, et al. Posterior segment ocular manifestations in patients with HLA-B27 segment ocular manifestations in patients with HLA B

26 Hayem F, Chacar S, Hayem G. Bartonella henselae infection mimicking systemic onset juvenile chronic arth tis in a 2.5 year old girl. 7 Rheumatol 1996;23:1263-5.

27 Wakefield D, Montanaro A, McCluskey PMC. Acute anterior uveitis and HLA B27. Surv Ophthalmol 1991;36:22332.

28 Ormerod D, Skolnick KA, Menesky MM, et al. Retinal and choroidal manifestations of cat-scratch disease. Ophthalmology 1998;105:1024-31.

29 Drancout M, Mainardi JL, Brouqui P, et al. Bartonella Rochalimaea) quintana endocarditis in three homeless men. N Engl f Med 1995;332:419-23.

30 Baggia S, Lyons JL, Barkhuizen A, et al. A novel model of bacterially-induced acute anterior uveitis in rats and the lack of effect from HLA B27 expression. $\mathcal{F}$ Invest Med 1997;45:295-301.

31 Blakenberg-Sprenkels SHD, Fielder M, Feltkamp TEW, et al. Antibodies to Klebsiella pneumoniae in Dutch patients with ankylosing spondylitis and acute anterior uveitis and to Proteus mirabilis in rheumatoid arthritis. 7 Rheumatol 1998;25:743-7.

32 Granfors K, Jalkananen S, van Essen R, et al. Yersinea antigens in synovial-fluid cells from patients with reactive arthritis. N Engl F Med 1989;26:216-21.

33 Warren K, Goldstein E, Hung VS, et al. Use of retinal biopsy to diagnose B henselae retinitis in an HIV-infected patient. Arch Ophthalmol 1998;116:937-40.

34 Kerkhoff FT, Bergmans AMC, van der Zee A, et al. Demonstration of Bartonella grahamii DNA in the ocular fluids of a patient with neuroretinitis. F Clin Microbiol 1999;37: 4034-8.

35 Uksila J, Toivanen P. HLA-B27 and bacteria. Clin Rheumatol 1996;15(Suppl 1):44-7.

36 Kapasi K, Inman RD. HLA B27 expression modulates gram negative bacterial invasion into transfected L cells. F Immunol 1992;148:3554-9.

37 Maurin M, Birtles RJ, Raoult D. Current knowledge of Bartonella species. Eur f Clin Microbiol Infect Dis 1997;16:487506. 